\title{
Reallocating attention during multiple object tracking
}

\author{
Justin M. Ericson • James C. Christensen
}

Published online: 3 April 2012

(C) Springer Science+Business Media, LLC (outside the USA) 2012

\begin{abstract}
Wolfe, Place, and Horowitz (Psychonomic Bulletin \& Review 14:344-349, 2007) found that participants were relatively unaffected by selecting and deselecting targets while performing a multiple object tracking task, such that maintaining tracking was possible for longer durations than the few seconds typically studied. Though this result was generally consistent with other findings on tracking duration (Franconeri, Jonathon, \& Scimeca Psychological Science 21:920-925, 2010), it was inconsistent with research involving cuing paradigms, specifically precues (Pylyshyn \& Annan Spatial Vision 19:485-504, 2006). In the present research, we broke down the addition and removal of targets into separate conditions and incorporated a simple performance model to evaluate the costs associated with the selection and deselection of moving targets. Across three experiments, we demonstrated evidence against a cost being associated with any shift in attention, but rather that varying the type of cue used for target deselection produces no additional cost to performance and that hysteresis effects are not induced by a reduction in tracking load.
\end{abstract}

Keywords Object-based attention · Selective attention

\section{Introduction}

Over 20 years of research on the visual tracking of multiple independently moving objects has led to some consensus

J. M. Ericson $(\bowtie) \cdot$ J. C. Christensen

Air Force Research Laboratory, Wright-Patterson Air Force Base, 2255 H Street B248,

Dayton, OH 45433, USA

e-mail: james.christensen@wpafb.af.mil

J. M. Ericson

Louisiana State University,

Baton Rouge, LA, USA regarding the mechanisms supporting this ability. Attention is deployed in parallel (Howe, Cohen, Pinto, \& Horowitz, 2010) to enhance targets and suppress distractors (Doran \& Hoffman, 2010), in a manner similar to on/center-off/ surround receptive fields (Koch \& Ullman, 1985; Pylyshyn, 2000; Walther et al., 2002). This is the mechanism discussed by Franconeri, Jonathon, and Scimeca (2010), supporting their argument that interference between attentional loci caused by close object spacing is the only limiting factor in multiple object tracking, although perceptual uncertainty may underlie many of these effects (Vul, Frank, Alvarez, \& Tenenbaum, 2009) and tracking can interfere with other tasks, such as maintaining object identity (Pylyshyn, 2004).

A key aspect of this proposed mechanism is that it is capable of arbitrary selection and deselection, which is the "shift problem" discussed by Koch and Ullman (1985). Within the context of multiple object tracking, relatively little is known about the processes of selecting and deselecting targets. Computational theories of visual attention (e.g., Bundesen, 1990; Itti \& Koch, 2001; Kazanovich \& Borisyuk, 2006) have indicated that some sort of cost in terms of time or accuracy should be associated with a shift in attention, due to the need to inhibit one locus and activate another. Similarly, if humans track multiple objects via grouping into a constructed superordinate object (Fehd \& Seiffert, 2008; Yantis, 1992), selection and deselection should require reforming the object and should incur some cost in performance. On the other hand, research on selective attention has suggested that the cost of reallocating attention may vary with cue compatibility; selecting a target that has been cued with a sudden visual onset (Yantis \& Jonides, 1990) should be less costly than deselecting a target cued in the same manner. Pylyshyn and Annan (2006) found exactly that in their study of static precues prior to a typical multiple object tracking task: Selecting the onset-cued set 
was faster and less error prone than selecting the complementary objects that were not onset-cued.

However, selecting and deselecting targets during tracking, as opposed to prior to tracking, has been found to produce little or no long-term impact on performance (Wolfe, Place, \& Horowitz, 2007). Wolfe et al. showed that participants were relatively unaffected by selecting and deselecting targets during tracking, and were thus capable of maintaining tracking for much longer periods of time than the few seconds typically studied. This is broadly consistent with the results of Franconeri et al. (2010), who found that tracking time is not inherently a significant contributor to performance decrements, but inconsistent with Pylyshyn and Annan's (2006) results using precues. On the one hand, precues may simply be processed differently than cues during tracking; on the other, there may be some cost of deselection, as in Pylyshyn and Annan's study, that is cancelled by a performance benefit for selection relative to tracking a fixed set. The continuous trials of Wolfe et al. did not enable separate analyses of selection and deselection. We therefore chose to examine their results in some detail by breaking down the selection and deselection of targets into separate conditions. As Wolfe et al. pointed out, it is difficult to compare performance under these circumstances, given the variation in the numbers of targets tracked. We will present a simple performance model that enables the comparison of actual performance when the target set is altered to what would be predicted had there been no cost of selection or deselection.

Multiple plausible hypotheses may explain the results of this comparison; we will address three distinct categories. (1) Computational models imply that there could be a cost of any shift in attention, such that performance in the shift conditions is consistently lower than that predicted by the fixed conditions. (2a) Pylyshyn and Annan's (2006) results suggested that interference between the onset cue and the action of deselection could result in decreased performance for deselection but no impact on selection. We would then predict that offsetting a target to cue deselection would eliminate this interference and improve performance. (2b) An alternative hypothesis leading to the same result is that the decrease in task demand that results from reducing the size of the tracked set may cause aftereffects or performance hysteresis, possibly due to depletion of the cognitive resources necessary for performing a demanding task (Kahneman, 1973; Krulewitz, Warm, \& Wohl, 1975; Matthews, 1986). (3) Finally, the results of Wolfe et al. (2007) suggest that there may be little or no observed cost of selecting and deselecting targets. This result could be obtained (3a) if reallocation is highly efficient, leading to no difference between predicted and actual performance, or (3b) if there were a relative benefit for selection and a similar cost for deselection, even after modeling out the probabilistic effects of variation in set size on observed performance. The benefit of selection would then be difficult to explain, though the relative cost of deselection could be explained by either of the second class of hypotheses.

We therefore chose to construct a version of the multiple object juggling task that Wolfe et al. (2007) introduced. Selection and deselection of targets were limited to a single event in each trial, thus isolating either of the two events in order to enable separate evaluation of performance differences. Three experiments are described in the present article. The first was intended to discriminate between the three categories of hypotheses presented above by comparing selecting and deselecting a target with predicted performance that was derived from tracking a fixed set. The results were somewhat mixed, providing evidence strongly against a cost for any shift in attention (1) or an offsetting of costs and benefits ( $3 b$ ), but not clearly discriminating between the remaining possibilities. In a second experiment we compared offset versus onset cues, which demonstrated no effect of the cue type on performance, and was thus inconsistent with cue interference (2a). The preceding studies consistently showed no difference between predicted and actual performance for deselection and selection, which supports Hypothesis 3a; a stronger test for the presence of performance hysteresis $(2 b)$ was conducted by repeating the first experiment under reduced overall set sizes. If hysteresis was the cause for decreased performance, smaller overall set sizes should reduce the impact of hysteresis, and thus performance should exceed predictions. Performance, in fact, was not significantly different from predictions and was collinear with that obtained using larger set sizes, despite the difference in the average numbers of targets tracked; low performers starting with large sets performed very similarly to high performers with small sets. Had hysteresis been a factor, we would have expected that a larger initial load would result in a greater decline in performance. The remaining hypothesis (3a) was borne out, that reallocation of attention under these conditions is surprisingly efficient and is accomplished without a significant performance cost.

\section{Experiment 1}

Method

Participants A group of 11 naïve personnel currently employed at Wright-Patterson Air Force Base in Dayton, Ohio, volunteered to participate in the study. The participants completed comprehensive written informed consent prior to the start of the experiment, and all reported normal or corrected-to-normal vision with no color blindness. Four of the participants were female and seven male, all between the ages of 20 and 35 years. 
Apparatus and stimuli The stimulus display and experimental control were managed using MATLAB version R2008a (The Mathworks, Inc., Natick, MA) and the Psychophysics Toolbox (Brainard, 1997; Pelli, 1997), running on a Dell PC with a 19-in. monitor running at a screen resolution of $1,280 \times 1,024$ and a $75-\mathrm{Hz}$ refresh rate. The display consisted of a white square frame that subtended $20.6^{\circ}$ of visual angle, defining the tracking area, and 10 identical white dots, each approximately $0.35^{\circ}$ of visual angle in diameter, on a black background. The dots moved at approximately $10^{\circ}$ per second in a linear trajectory, bouncing off the edges of the display frame and occluding each other while in motion. If a switch in the tracked set occurred, it was cued by a color change in the dot to be switched, to either an equiluminant $\left(11.81 \mathrm{~cd} / \mathrm{m}^{2}\right)$ green (add) or red (remove), presented in addition to an accompanying tone to alert the observer that a change was occurring.

Procedure At the start of each trial, the 10 white, circular dots were displayed on the screen within the defined display frame. With the display static, white circles surrounding a dot at the start of the trial were used to cue the participant as to which of the objects were to be tracked; these cues remained on the screen for $5 \mathrm{~s}$. After this period the cues were removed, and a tone sounded to alert observers to the start of the motion sequence. Following the conclusion of the motion sequence, the participants identified the dots that they believed had previously been cued as targets by directing the mouse pointer over each dot and pressing the space bar. Performance feedback was given through the use of high and low tones following each space bar press; high tones indicated correct responses, and low tones indicated incorrect responses.

The experiment consisted of six conditions. Each condition was presented four times per block in a random order. Four blocks were run, resulting in 16 repetitions per condition and 96 trials total for the complete experiment. Following each trial was a break of $500 \mathrm{~ms}$, and at the end of each block performance feedback was given in percentages correct. The first two conditions were 20 -s tracking trials, in which participants tracked either three or four targets out of the 10 dots displayed for $20 \mathrm{~s}$. The second two conditions were 10-s conditions, in which participants again tracked either three or four targets, but for only half of the duration of the 20-s tracking trials.

The final two conditions were switch conditions, in which either a new target dot was added to a tracked set of three $(3+)$ or a current target dot was removed from a tracked set of four (4-). In these conditions (see Fig. 1 for the time course), a participant was presented with three $(3+)$ or four targets (4-) at the start of the trial. The trial sequence was similar to that of the long tracking trials, except that at the midpoint of the tracking

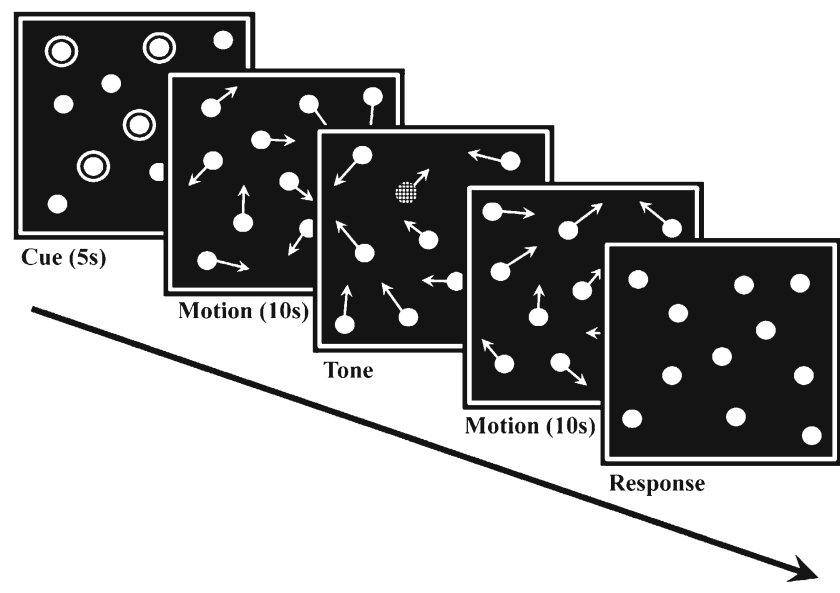

Fig. 1 Trial sequence for the switched trials. The crosshatching on the dot in the center panel indicates where a color change took place in the study. The color change to red indicated that participants should remove that target from the tracked set, while a change to green indicated that the cued nontarget should be added to the tracked set. Unswitched trials simply featured continuous motion with no color cues

duration (10 s), participants were cued via a color change to green (add) or red (remove), in addition to the nonspecific auditory switch cue. The color change lasted for $500 \mathrm{~ms}$, after which time the dot returned to white. It should be noted that an "add" cue would only occur for trials that started with three targets, and "remove" cues would only occur for trials that started with four targets, although the random intermixing of the other trial types ensured that participants could not predict the trial type on the basis of the initial number of dots tracked.

\section{Results}

All proportions correct and numbers of dots effectively tracked are based on $m$ scores (Pylyshyn \& Annan, 2006), which correct for differences in guessing probabilities as the number of targets changes. The basic results are presented in Fig. 2. The effect of removing a dot appears to be equal and opposite to the effect of adding a dot, despite the trials being essentially mirror images of each other. However, because these trials were performed continuously, the performance following addition or removal was dependent on performance in the first half of a trial; larger drops in performance in the first half would limit the maximum overall performance in the trial. In the switch condition in which a dot was removed, two scenarios are possible: that the removed dot had been lost from tracking prior to the switch by the participant, leading to no particular performance penalty, or that the removed dot was one that the participant was still tracking, leading to a large negative effect on overall performance. 


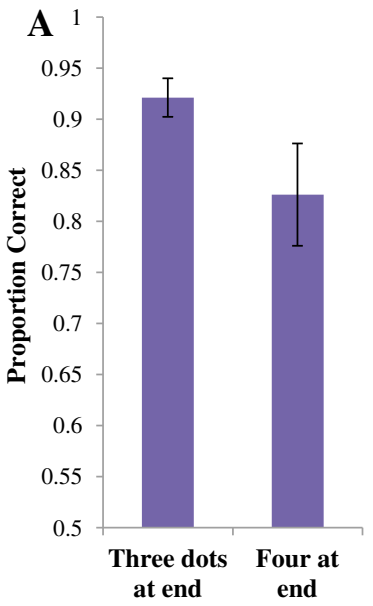

Fig. 2 Mean proportions correct (based on corrected $m$ scores) in all six conditions of Experiment 1. The left panel shows the results of the unswitched 10-s conditions. The right panel shows all of the 20-s conditions, including model predictions for the switched conditions. The 3+ and 4- switched conditions are significantly different from each other, $t(10)=10.71, p<.001$ (Bonferroni corrected for three

To address these concerns and to aid in interpreting the results, a simple model of tracking performance in this task was constructed with two key assumptions: first, that the probability of a dot being lost from the tracked set at any given time is a function of the number of dots being tracked at that time, and second, that switches in the tracked set were accomplished with no performance penalty. This second assumption enables the comparison of predicted performance with the actual results to determine the effects of adding and removing dots. This approach does not attempt to explain the role of attention in performing the task, but rather to provide accurate comparisons that will constrain the construction of models such as FINST (Pylyshyn \& Storm, 1988), object files (Kahneman, Treisman, \& Gibbs, 1992), or the neural network model of Kazanovich and Borisyuk (2006).

The first assumption is an extension of Pylyshyn and Storm's (1988) original experiments demonstrating that increasing tracking load led to more targets being lost over a fixed interval. Rather than considering each trial as a discrete event, we could instead consider a trial to be a contiguous series of tracking periods, with tracking load decreasing over time; as tracking load decreases, the ability to maintain that load should increase and lead to an asymptote in performance over time. This idea is supported by the work of Shim, Alvarez, and Jiang (2008), who proposed that a significant and perhaps primary source of errors in this task is interference caused by close spacing (Franconeri et al., 2010) or near-miss events. Similarly, Vul et al. (2009) pointed out that close spacing increases perceptual uncertainty and can explain the rise in errors. With our fixed display size, increasing the proportion of targets necessarily comparisons), and the difference is similar to the performance difference between tracking three and four dots in the unswitched conditions. The model predictions are not significantly different from the actual performance for the $3+$ condition, $t(10)=1.4$, and the 4 condition, $t(10)=0.68$

would increase target density and the frequency of near-miss events involving a target; however, even during a trial, as tracking is lost on individual targets, density decreases, as does the frequency of near misses. For these reasons, the rate at which targets are lost was estimated separately for the first and second halves of our 20-s-long trials. Likewise, the proportion of errors increases as trial length increases and tracked set size is held constant (see Oksama \& Hyönä, 2004, for set sizes less than six; they suggested that six in the set might have triggered a change in response strategy and/or only tracking a subgroup). The assumption that performance is a function of the number of dots tracked was internally checked by comparing two different trial segments with comparable numbers of initial targets. The average number of targets lost in the 10 -s, three-dot condition was compared with the number of targets lost in the second half of the 20-s, four-dot condition. On the basis of performance in the 10-s, four-dot condition, we estimated that the second half of the 20 -s, four-dot condition would start with an average of 3.3 dots. The number of targets lost in the second half was then estimated by subtracting the mean number correct (corrected for guessing) in the 20 -s trial from the same mean in the 10 -s trial. This value was not significantly different from the number of targets lost in the 10-s, three-dot condition: For the 10-s, three-dot condition, a mean of $0.24 \pm 0.11$ (.95 confidence interval [CI]; note that all subsequent error bounds will be expressed as the $.95 \mathrm{CI}$ as well) dots were lost, while in the second half of the fourdot condition, $0.35 \pm 0.17$ dots were lost, $t(10)=1.3$, n.s. In contrast, comparing the 10 -s, four-dot condition with the 10 $\mathrm{s}$, three-dot condition resulted in significantly fewer dots being lost in the three-dot condition, $t(10)=2.72, p<.05$ 
(Bonferroni corrected), replicating Pylshyn and Storm's result.

For the switched condition in which a dot was added $(3+)$, performance was predicted by starting with the number of dots tracked successfully on the 10-s, three-dot trial, adding one for the added dot (assuming perfect uptake), and then using the estimated slope for the second half of the 20$\mathrm{s}$, four-dot trial, as calculated by subtracting the difference between the 20-s, four-dot trial and the 10-s, four-dot trial.

Predicting performance in the switched condition in which a dot was removed (4-) was similar but was complicated by the probability that a removed dot might or might not be tracked at the time of removal. To account for this probability, the error distributions for each participant in the 10 -s, four-dot condition were used as an estimate of what was being tracked immediately prior to removing the dot, as follows in Eq. 1:

$\sum_{i=1}^{4} \frac{-p\left(c_{i}\right) \times i}{4}$

where $i$ is the number of correct responses and $p\left(c_{i}\right)$ is the proportion of trials with exactly $i$ correct responses, following a correction for guessing developed by Hulleman (2005); note that the overall form was inspired by Eq. 5 from Pylyshyn and Storm (1988). The results of this equation will be some fraction of -1 , to be subtracted from the number of dots tracked at that point; if a participant performed perfectly in the 10 -s, four-dot condition, the predicted effect would be to subtract 1 . Lower performance would decrease the negative impact, as the probability that the removed dot had already been lost prior to the switch would increase. The rest of the prediction for the 4- condition would then be calculated by subtracting this correction from performance in the 10-s, four-dot condition and subsequently subtracting the difference between the 20 -s, threedot trial and the 10-s, three-dot trial. The approach is presented with example data in Fig. 3.

The results of regressing the predicted performance against actual performance in the switched conditions are presented in Fig. 4. In both the 3+ and 4-conditions, the proportions of variance explained by the model are relatively high, at $R^{2}=.90$ and .79 , respectively. For the $3+$ condition, the slope and intercept are not significantly different from 1 and 0 , respectively: slope $=1.01 \pm 0.22$ and intercept $=-0.12 \pm 0.81$. In the $4-$ condition, the slope $(0.48 \pm$ 0.69 ) is marginally different from 1 , while the intercept $(1.18 \pm 0.34)$ is significantly greater than 0 . The high observed $R^{2}$ values are not simply a consequence of using one portion of each participant's data to predict another; regressing performance in the unswitched conditions against the switched conditions ending with the same number of dots resulted in $R^{2} \approx .7$. The increased variance accounted for by

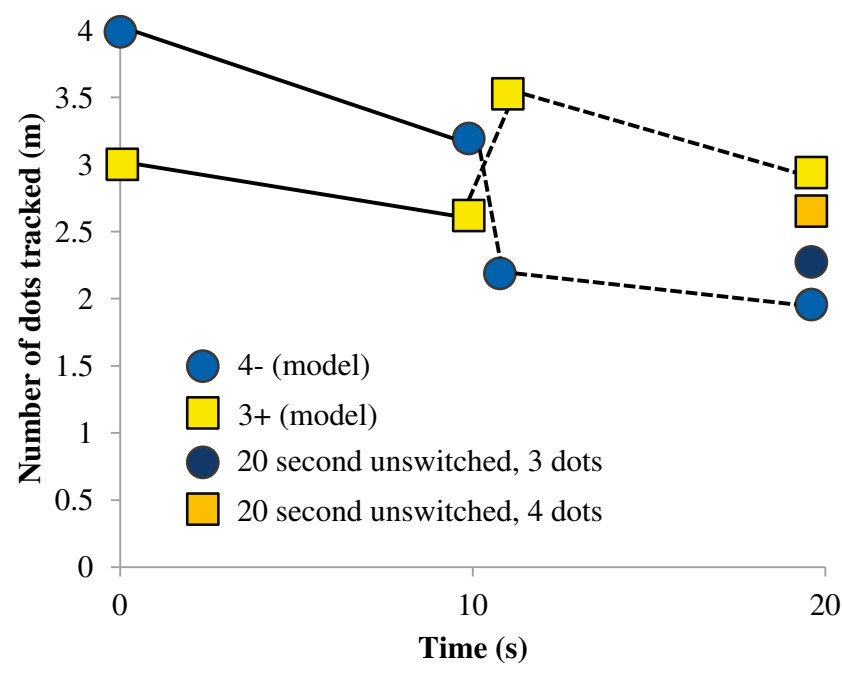

Fig. 3 Example of how performance predictions were generated for the switched conditions on the basis of data from the fixed conditions. The values used are drawn from one selected participant. Solid lines are drawn between actual performance points, and dashed lines indicate that the following point is predicted. The loss of tracking on individual objects over time was approximated via piecewise linear fits between the three data points available: all targets at the beginning, and then performance in the 10- and 20-s unswitched conditions. The impact of the switch was calculated either by adding a whole dot (in the $3+$ condition) or subtracting a fraction of one that depended on the performance immediately prior to the switch (in the 4- condition)

the model likely reflects differences between the observers in both asymptotic performance (consistent with Oksama \& Hyönä, 2004) and the rate of object loss. The relatively good fit in the $3+$ condition supports the assumptions of the model, suggesting that the performance penalty of adding

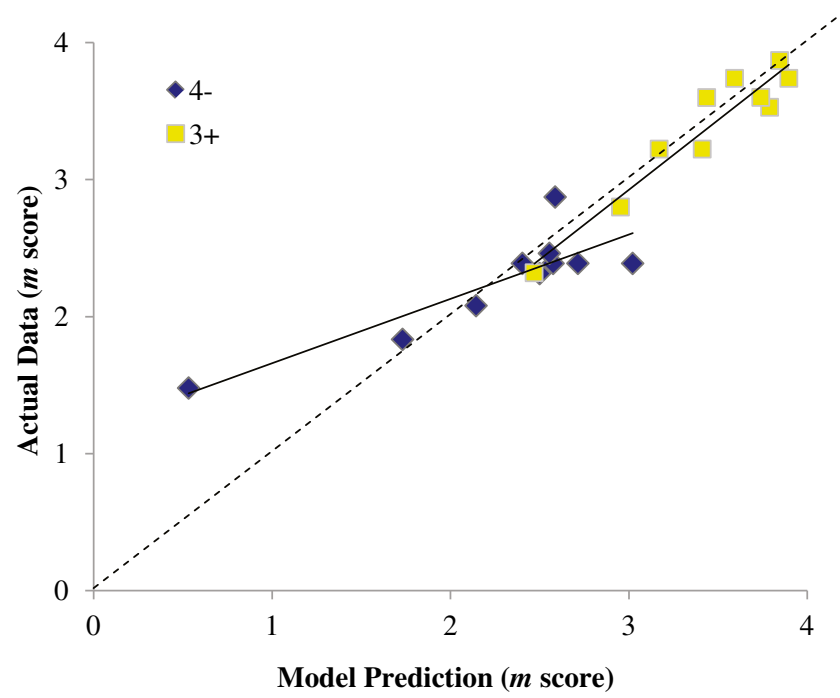

Fig. 4 Linear regression of predicted against actual performance for the switched conditions of Experiment 1. Each plotted symbol represents one participant in the appropriate condition. In both switched conditions, the proportion of variance in the actual performance accounted for exceeds .9; however, in the 4 - condition the model prediction appears to be off by a scaling factor 
a dot was small or nonexistent. The prediction for the 4condition was accurate only at intermediate levels of individual performance. As will be seen from the replicated conditions in the next two experiments, however, this result may be an outlier, and more generally the model prediction is accurate for the 4- condition.

In light of these results, it is possible to strongly reject the first class of hypotheses, wherein any shift in the tracked set reduces performance. The model prediction and actual results for the $3+$ condition are highly similar and do not reveal a positive or negative impact of the switch. The lack of any performance improvement in this condition also argues against offsetting costs and benefits for selection and deselection (Hypothesis $3 b$ ) that sum to no overall difference, as was observed in Wolfe et al. (2007). The results obtained by comparing the model predictions and performance in the 4- condition are somewhat ambiguous on the basis of this study alone; there may or may not be a negative impact of deselection, and thus it is difficult to discriminate between the remaining hypotheses. We therefore conducted a second experiment in which we directly tested the possibility that cue interference leads to decreased performance in the deselection condition (2a), while in a third experiment we tested the possibility that hysteresis caused a decrease in performance (2b).

\section{Experiment 2}

\section{Method}

Participants A group of 15 undergraduate students at Louisiana State University participated in order to receive course credit. The participants completed comprehensive written informed consent prior to the start of the experiment, and all reported normal or corrected-to-normal vision with no color blindness. Eleven of the participants were female and four male, all between the ages of 18 and 24 years.

Apparatus and stimuli The stimuli were transferred to an Apple iMac computer with a 21.5 -in. LCD display, running at a resolution of $1,024 \times 768$. The experiment was managed using MATLAB R2008b (The Mathworks, Inc., Natick, MA) and the Psychophysics Toolbox (Brainard, 1997; Pelli, 1997). Experimental controls, such as the display parameters and durations, were identical to those of Experiment 1, except that two different cues were used to cue the addition or removal of a dot. In addition to the color cue used in Experiment 1, in one of the new conditions a dot to be removed simply disappeared (a sudden offset), while in the second new condition a dot was color cued and then disappeared (a blinked offset). Any dot removed in this way was added back to maintain the total set size by splitting a new dot from a randomly selected nontarget, as if an occlusion had taken place.

Procedure Five of the conditions from Experiment 1 were repeated: specifically, the 20-s, four-dot and three-dot trials; the 10 -s, four-dot and three-dot trials; and the 4-switched condition, in which a dot was removed from an initial set of four tracked dots. The two new conditions consisted of removing a target from the tracked set without a color cue (offset) or by cuing the target to be removed and then having it disappear from the display (blink). As before, participants selected the dots that they believed to be targets. Each of the seven conditions was presented four times per block in random order. Four blocks were run, resulting in 16 repetitions per condition and 112 trials total for the complete experiment. To facilitate data collection in a computing lab environment, feedback was no longer given via tones, but rather the word "correct" or "incorrect" was displayed above the display frame after each appropriate response.

\section{Results}

The proportions correct for each of the three removal conditions are shown in Fig. 5. There was no significant effect of cue type, $F(2,24)=0.64$, n.s. The overall proportions correct are consistent with that observed for the removal condition in Experiment 1, and performance in the remaining conditions (used to generate model predictions) was likewise consistent with that in Experiment 1.

Applying the performance model as in Experiment 1, we regressed the model predictions for each of these conditions against actual performance (see Fig. 6); note that the model predictions are identical for all conditions, as the input data

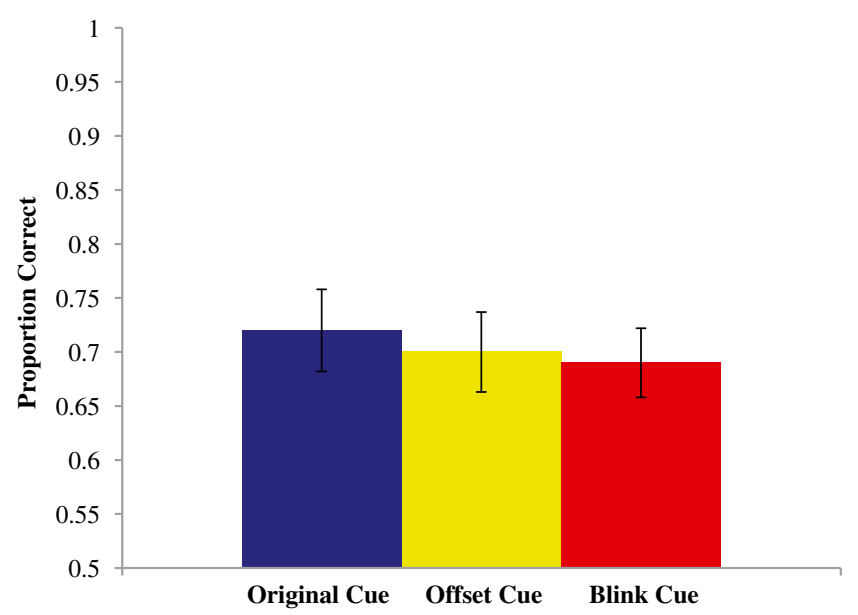

Fig. 5 Mean proportions correct (based on $m$ scores) for the switch conditions in Experiment 2. There were no significant differences for different cue types when removing a target from the tracked set 


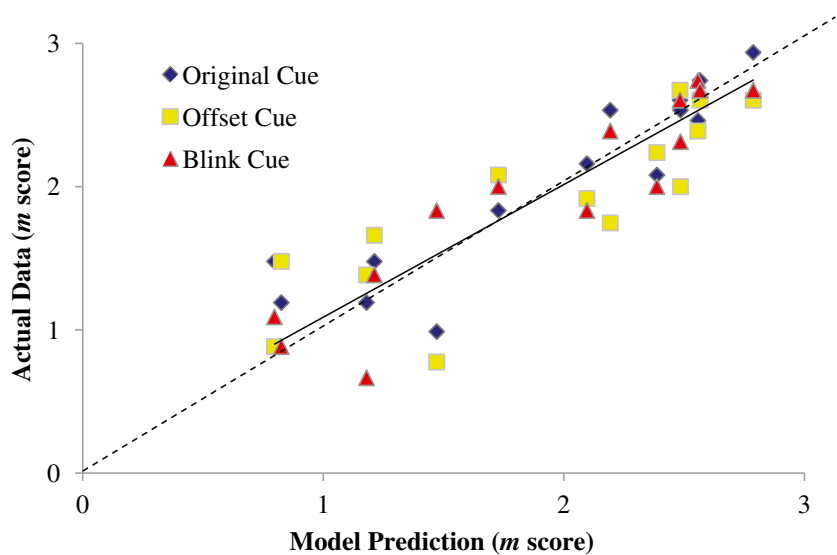

Fig. 6 Regression of predicted against actual performance for the different cue conditions of Experiment 2. The aggregate $R^{2}$ for the conditions equaled .79

are drawn from the same fixed-target-set conditions. As would be expected from the proportions correct, the resulting regression equations are not significantly different from each other. After collapsing across cue types, the combined regression equation resulted in an $R^{2}$ similar to that in Experiment 1, at .79, while the slope was $0.84 \pm 0.3$, greater than that observed in Experiment $1(0.48 \pm 0.69)$ and not significantly different from 1 . The intercept was similarly close to 0 , albeit unstable, at $0.3 \pm 0.79$. For comparison, regressing performance in the comparable unswitched condition against the switched conditions resulted in $R^{2} \approx .4$. These data are overall noisier than those in Experiment 1, despite the increased number of participants, and this could have been caused by the change in the participant population.

In contrast to Pylyshyn and Annan (2006), manipulating the cue type did not result in significant differences in performance for the removal condition. This may well have been because, unlike in their study, only deselection was required, and not selection of the complementary set. Nevertheless, this refutes the cue interference hypothesis (2b), under which deselection of an onset-cued target results in lower performance. While these data are noisier than Experiment 1, they do not exhibit a regression slope less than 1, as was observed in the original deselection condition. To anticipate the results of Experiment 3, which primarily tested the hysteresis hypothesis, a slope of 1 and intercept 0 were consistent across the replications contained in Experiments 2 and 3. A valid concern, however, is that the additional noise in these experiments may have rendered them underpowered to detect a slope less than 1; following Experiment 3, we will present a posthoc power analysis that suggests that they were of adequate power to discriminate a slope of 1 from a slope of 0.5, as observed in Experiment 1.

\section{Experiment 3}

Method

Participants A group of 14 undergraduate students at Louisiana State University participated in order to receive course credit. The participants completed comprehensive written informed consent prior to the start of the experiment, and all reported normal or corrected-to-normal vision with no color blindness. Ten of the participants were female and four male, all between the ages of 18 and 22 years.

Apparatus and stimuli The stimulus and experimental controls were identical to those of Experiment 2.

Procedure Three of the conditions from Experiment 2 were repeated-specifically, the 20-s, three-dot trials; the 10-s, four-dot trials; and the 4- switched condition, in which a dot was removed from an initial set of four tracked dots. Three new conditions were also included, produced simply by reducing the number of dots tracked for each condition. This resulted in 20-s, two-dot trials; 10 -s, three-dot trials; and a new 3 - switched condition, in which a dot was cued for removal from an initial set of three tracked dots. On the basis of pilot results, performance was at ceiling in a 10-s-long two-dot trial; as a result, this condition was dropped and ceiling performance was assumed for modeling purposes. Again, the participants were to select the dots that they believed to be targets. Each of the six conditions was presented four times per block in random order. Four blocks were run, resulting in 16 repetitions per condition and 96 total trials in the experiment.

\section{Results}

The results from the four 20-s conditions are presented in Fig. 7. A repeated measures ANOVA revealed significant main effects of number of targets, $F(1,13)=25.5, p<.01$, and of switched versus unswitched trials, $F(1,13)=18.7, p<.01$. There was no significant interaction, $F(1,13)=.180, p=.678)$.

The performance model from Experiment 1 was adapted to this data set by replacing all of the conditions with their reduced equivalents. Starting with the number of targets effectively tracked in the 10 -s, three-dot trials, we subtracted a fraction of 1 (with 3 in the place of 4 in Eq. 1) and then subtracted the estimate for target loss in the last half: the 20$\mathrm{s}$, two-dot condition minus the 10-s, two-dot condition. Performance in the 10-s, two-dot condition was assumed to be at ceiling on the basis of pilot data; in the full study, the 20-s, two-dot condition was close to ceiling, as well. Regressing the model predictions against actual performance in the 4- switched condition resulted in a slope of $0.92 \pm 0.22$ and an intercept of $0.07 \pm 0.49, R^{2}=.88$. The $3-$ switched condition resulted in a slope of $0.70 \pm 0.41$ and an 


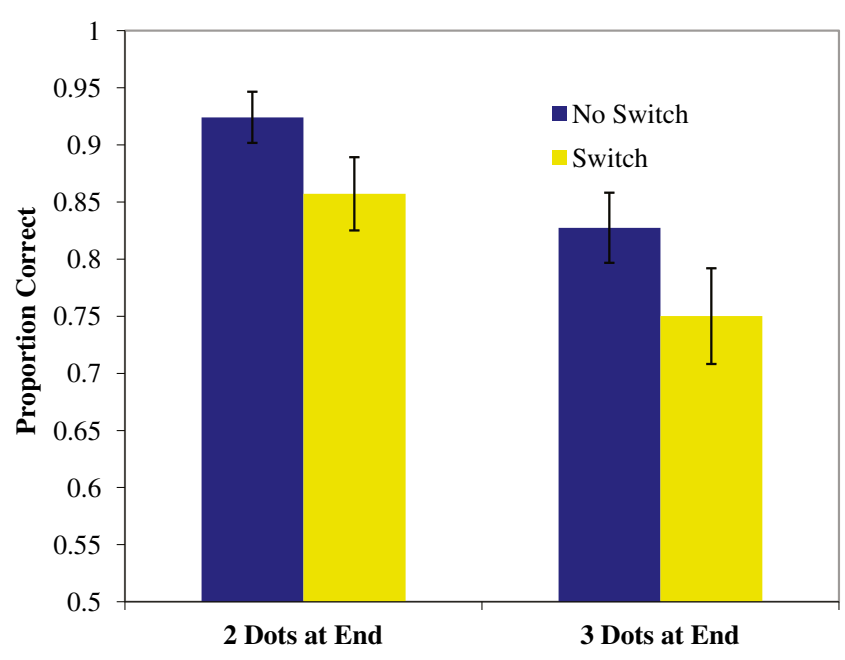

Fig. 7 Mean proportions correct for the 20-s trials in Experiment 3. Removing a dot from the tracked set appears to negatively affect performance, regardless of set size

intercept of $0.52 \pm 0.54, R^{2}=.70$ (Fig. 8). Performance in the 3-condition was essentially continuous with that observed in the 4- condition, which suggests that a lowerperforming participant in the 4- condition was equivalent to a higher-performing participant in the 3-condition; thus, there was no effect of having started with more targets. As the model was set up with an assumption of zero cost or benefit from changing the tracked set, the close fits in both conditions suggests that hysteresis was unlikely.

As discussed earlier, the regression slopes in the identical 4- conditions from Experiments 2 and 3 were greater than that found in Experiment 1 (approximately 1 vs. 0.5), as were the overall variances of the data. This raises the possibility that the true value may be closer to 0.5 ; a post hoc power analysis was performed ( $\mathrm{G}^{*}$ Power; Faul, Erdfelder, Lang, \& Buchner, 2007) that aggregated the 4- data from Experiments 2 and 3, totaling 29 participants. In this sample,

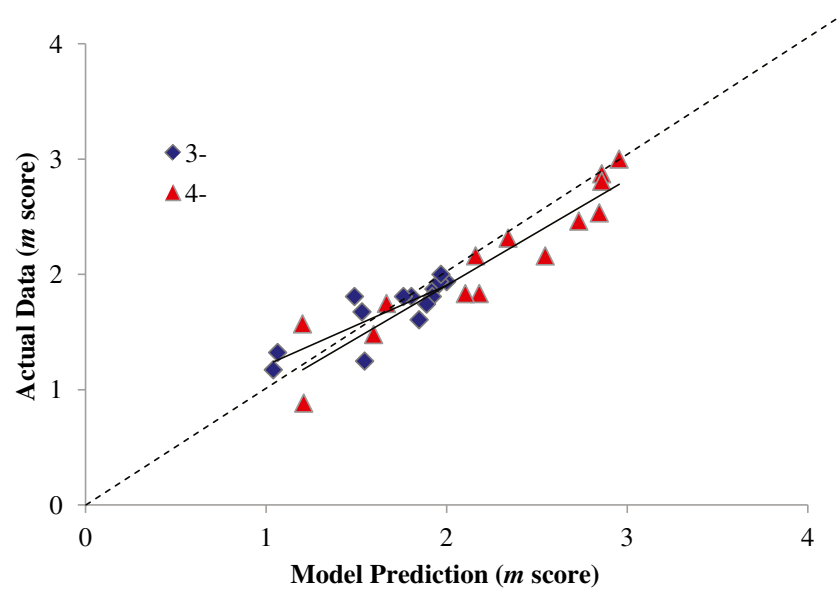

Fig. 8 Regression of predicted against actual performance for the different set sizes of Experiment 3 our observed power $(1-\beta$ at $\alpha=.05)$ was $\sim .68$; while this result is not conclusive, it is evidence that our data are sufficient to discount the slope from Experiment 1 as an outlier.

\section{Discussion}

In light of the results of these three experiments, it is now possible to reexamine the results reported in Wolfe et al. (2007). The continuous addition and removal of single targets in their study resulted in very little effect on performance; from the results presented in Experiment 1, it seems likely that their results were a consequence of the apparent costs and benefits of addition and removal (Fig. 2) canceling each other out over time. From the experiments presented above, these costs and benefits do not appear to be intrinsic to the switch in tracking, but rather reflect the consequences of differing performance as a function of target set size and the probabilistic effects associated with deselection. Because our tracking space and total number of objects were fixed, target-target spatial proximity interference varied directly with changes in tracking set size. Our results therefore are consistent with Franconeri et al. (2010), in that the momentary number of targets tracked was most predictive of performance, though any other such theory in which immediate tracking load determines the likelihood of losing tracking on a dot would also predict this result.

Three categories of hypotheses regarding the reallocation of attention during multiple object tracking were considered in designing these studies: The first is that any change decreases performance, the second that only deselection decreases performance, and the third that selection and deselection have no effect on performance. The first and second hypotheses were unlikely given Wolfe et al.'s (2007) results, but they had been motivated by related work (Fehd \& Seiffert, 2008; Pylyshyn \& Annan, 2006; Yantis, 1992) and were still necessary to test. Our Experiment 1 results are not explainable by the first category, as there was no decrease in performance, whether model-predicted or raw, when selecting an additional dot. Likewise, the lack of a difference between the model-predicted and actual performance in that condition suggests that selecting an additional dot provides no intrinsic benefit, which is not consistent with the offsetting-increases-and-decreases version of the third category of hypotheses (3b). On this basis, the common strategy of constructing a polygon of the target objects (Fehd \& Seiffert, 2008; Yantis, 1992) is not significantly impacted by adding or removing a vertex.

One version of the second class of hypotheses was tested in Experiment 2: that the cue interfered with deselection but not selection (2a). Varying the cue type used for deselection did not result in significant differences in performance. This 
is inconsistent with the cue interference hypothesis, under which deselection of an onset-cued target would result in lower performance as compared to deselection of an offsetcued target. Finally, Experiment 3 tested the hypothesis that performance hysteresis from high tracking load may cause an excess decline in performance (number of targets tracked) for the deselection condition (2b) as compared to keeping a fixed tracking set. High tracking load could result in decreased performance for at least two reasons: It increases the number of near-miss events that can cause a loss of tracking on a target, but it also produces higher cognitive workload, which has in some cases been found to produce an undershoot in performance, or hysteresis (Krulewitz et al., 1975), following a switch from higher to lower load. If hysteresis were a significant contributor to decreased performance, it should have been attenuated by the overall decrease in tracking load. However, the results show that the observed performance decrements were identical in both conditions and that the relationship between model predictions and performance was continuous between the two. This suggests that hysteresis was not a significant factor, which leaves Hypothesis 3a, that selection and deselection - at least of single targets - are accomplished efficiently, and therefore with no performance impact beyond the additional factors built into the performance model.

The performance model introduced here is not intended to explain the mechanisms that humans use to accomplish multiple object tracking, but rather to provide a statistical prediction under conditions of varying tracking load. This was necessary to enable comparisons across conditions within our study, but the model also makes testable predictions that are supported by the data presented here. One prediction central to the model is that tracking performance is independent of task history: The model would be disproven if it could be shown that tracking history influences performance above and beyond estimates of immediate tracking load. Another prediction is that performance will follow exponential decay when averaged across trials; the binary nature of performance scoring in this task results in discrete performance on individual trials, although there is evidence that visual working memory representations terminate in a sudden and complete fashion (Zhang \& Luck, 2009). This prediction is consistent with Shim et al. (Shim et al. 2008): If each near-miss event has a discrete probability of causing a loss of tracking on the involved target or targets, then averaging a reasonable number of stochastic trials will produce an exponential decay function for number of targets tracked. The present experiments did not result in enough data from trials of varying lengths to reliably fit decay functions that would allow variance in the asymptote, but such fits would be informative about individual differences in decay rates and the asymptotic level of performance that could be sustained over very long tracking durations.
Informal curve-fitting using a least-squared-error criterion of our data led to a mean asymptotic value of 2.07 dots, which is consistent with the modal effective number of dots tracked in Oksama and Hyönä (2004) in all conditions with tracking durations greater than $5 \mathrm{~s}$. This should not be interpreted as a generic value for minimal tracking performance, as the observed minimum would vary as a function of the tracking task parameters (Alvarez \& Cavanagh, 2004; Alvarez \& Franconeri, 2007). The exponential-decay view is consistent with Franconeri et al. (2010); time is a significant factor only because of the accumulation of events that can cause a loss of tracking on target.

Beyond the differences noted between Experiment 2 and Pylyshyn and Annan's (2006) results, the lack of an effect of cue type in Experiment 2 suggests that comparatively little interference takes place between maintaining tracking and processing a single sudden-onset cue, partially addressing Doran and Hoffman's (2010) comments about the potential interference created by probes in probe-dot detection tasks conducted within multiple object tracking (e.g., Drew, McCollough, Horowitz, \& Vogel, 2009; Pylyshyn, 2006). While this result is not conclusive when extended to probedot methods, it is suggestive that the sudden onset of the probe does not interfere specifically with tracking performance, although in that context some performance effects may still be due to the dual-task nature of the method.

In conclusion, we support and extend the results of Wolfe et al. (2007): When multiple object tracking is studied in a context more consistent with real-world performance, tracking is found to be possible over longer durations than are normally studied, and is surprisingly robust to additions and removals from the tracked set.

Acknowledgments This research was funded by the Air Force Research Laboratory; funding to J.M.E. was administered by the Consortium Research Fellows Program. The authors wish to thank Melissa Beck for helpful insight and support of the study, and three anonymous reviewers for their assistance in improving the manuscript.

\section{References}

Alvarez, G. A., \& Cavanagh, P. (2004). The capacity of visual shortterm memory is set both by visual information load and by number of objects. Psychological Science, 15, 106-111. doi:10.1111/j.0963-7214.2004.01502006.x

Alvarez, G. A., \& Franconeri, S. L. (2007). How many objects can you track? Evidence for a resource-limited attentive tracking mechanism. Journal of Vision, 7(13), 14:1-10. doi:10.1167/7.13.14

Brainard, D. H. (1997). The Psychophysics Toolbox. Spatial Vision, 10, 433-436. doi:10.1163/156856897X00357

Bundesen, C. (1990). A theory of visual attention. Psychological Review, 97, 523-547. doi:10.1037/0033-295X.97.4.523

Doran, M. M., \& Hoffman, J. E. (2010). The role of visual attention in multiple object tracking: Evidence from ERPs. Attention, Perception, \& Psychophysics, 72, 32-52. 
Drew, T., McCollough, A. W., Horowitz, T. S., \& Vogel, E. K. (2009). Attentional enhancement during multiple-object tracking. Psychonomic Bulletin \& Review, 16, 411-417.

Faul, F., Erdfelder, E., Lang, A.-G., \& Buchner, A. (2007). G*Power 3: A flexible statistical power analysis program for the social, behavioral, and biomedical sciences. Behavior Research Methods, 39, 175-191. doi:10.3758/BF03193146

Fehd, H. M., \& Seiffert, A. E. (2008). Eye movements during multiple object tracking: Where do participants look? Cognition, 108, 201-209.

Franconeri, S. L., Jonathon, S. V., \& Scimeca, J. M. (2010). Tracking multiple objects is limited only by object spacing, not by speed, time, or capacity. Psychological Science, 21, 920-925.

Howe, P. D., Cohen, M. A., Pinto, Y., \& Horowitz, T. S. (2010). Distinguishing between parallel and serial accounts of multiple object tracking. Journal of Vision, 10(8), 11:1-20. doi:10.1167/ 10.8 .11

Hulleman, J. (2005). The mathematics of multiple-object tracking: From proportions correct to the number of items tracked. Vision Research, 45, 2298-2309.

Itti, L., \& Koch, C. (2001). Computational modeling of visual attention. Nature Reviews Neuroscience, 2, 194-203. doi:10.1038/35058500

Kahneman, D. (1973). Attention and effort. Englewood Cliffs, NJ: Prentice Hall.

Kahneman, D., Treisman, A. M., \& Gibbs, B. J. (1992). The reviewing of object files: Object-specific integration of information. Cognitive Psychology, 24, 175-219. doi:10.1016/0010-0285(92)90007-O

Kazanovich, Y., \& Borisyuk, R. (2006). An oscillatory neural model of multiple object tracking. Neural Computation, 18, 1413-1440.

Koch, C., \& Ullman, S. (1985). Shifts in selective visual attention: Towards the underlying neural circuitry. Human Neurobiology, 4, 219-227.

Krulewitz, J. E., Warm, J. S., \& Wohl, T. H. (1975). Effects of shifts in the rate of repetitive stimulation on sustained attention. Perception \& Psychophysics, 18, 245-249.

Matthews, M. L. (1986). The influence of visual workload history on visual performance. Human Factors, 28, 623-632.

Oksama, L., \& Hyönä, J. (2004). Is multiple object tracking carried out automatically by an early vision mechanism independent of higher-order cognition? An individual difference approach. Visual Cognition, 11, 631-671.
Pelli, D. G. (1997). The VideoToolbox software for visual psychophysics: Transforming numbers into movies. Spatial Vision, 10, 437 442. doi:10.1163/156856897X00366

Pylyshyn, Z. W. (2000). Situating vision in the world. Trends in Cognitive Sciences, 4, 197-207. doi:10.1016/S1364-6613(00)01477-7

Pylyshyn, Z. W. (2004). Some puzzling findings in multiple object tracking: I. Tracking without keeping track of object identities. Visual Cognition, 11, 801-822.

Pylyshyn, Z. W. (2006). Some puzzling findings in multiple object tracking (MOT): II. Inhibition of moving nontargets. Visual Cognition, 14, 175-198.

Pylyshyn, Z. W., \& Annan, V. (2006). Dynamics of target selection in multiple object tracking (MOT). Spatial Vision, 19, 485-504.

Pylyshyn, Z. W., \& Storm, R. W. (1988). Tracking multiple independent targets: Evidence for a parallel tracking mechanism. Spatial Vision, 3, 179-197. doi:10.1163/156856888X00122

Shim, W., Alvarez, G. A., \& Jiang, Y. (2008). Spatial separation between targets constrains maintenance of attention on multiple objects. Psychonomic Bulletin \& Review, 15, 390-397.

Vul, E., Frank, M., Alvarez, G., \& Tenenbaum, J. (2009). Explaining human multiple object tracking as resource-constrained approximate inference in a dynamic probabilistic model. In Y. Bengio, D. Shuurmans, J. Lafferty, C. K. I. Williams, \& A. Culotta (Eds.), Advances in neural information processing systems 22 (pp. 19551963). Cambridge, MA: MIT Press.

Walther, D., Itti, L., Riesenhuber, M., Poggio, T., \& Koch, C. (2002). Attentional selection for object recognition-A gentle way. Lecture Notes in Computer Science, 2525, 472-479.

Wolfe, J. M., Place, S. S., \& Horowitz, T. S. (2007). Multiple object juggling: Changing what is tracked during extended multiple object tracking. Psychonomic Bulletin \& Review, 14, 344-349. doi:10.3758/BF03194075

Yantis, S. (1992). Multielement visual tracking: Attention and perceptual organization. Cognitive Psychology, 24, 295-340.

Yantis, S., \& Jonides, J. (1990). Abrupt visual onsets and selective attention: Voluntary versus automatic allocation. Journal of Experimental Psychology: Human Perception and Performance, 16, 121-134. doi:10.1037/0096-1523.16.1.121

Zhang, W., \& Luck, S. J. (2009). Sudden death and gradual decay in visual working memory. Psychological Science, 20, 423-428. doi:10.1111/j.1467-9280.2009.02322.x 